\title{
Secular period decreasing of 17 detached chromospherically active binaries
}

\author{
C.Q.Luo ${ }^{1} \dagger$, Y.P. Luo $^{1}$, X.B. Zhang ${ }^{2}$, L.C. Deng, ${ }^{2}$ \\ Z.Q. Luo ${ }^{1}$, S.Z. Yang ${ }^{1}$ \\ ${ }^{1}$ Institute of Theoretical Physics, China West Normal University, IN Nanchong Sichuan, \\ 637002,China \\ email: changqingluo@126.com; luoyangping789@163.com; zqluo@tom.com; \\ szyangcwnu@126.com \\ ${ }^{2}$ National Astronomical Observatories, Chinese Academy of Sciences, IN Beijing, 100012, \\ China \\ email: xzhang@bao.ac.cn; licai@bao.ac.cn.
}

\begin{abstract}
The long-term orbital period changes of detached chromospheric active binaries were surveyed. 17 of such systems are found to be undergoing secular period decreasing with the rates $(\mathrm{dP} / \mathrm{dt})$ of $-3.05 \times 10^{-9}$ to $-3.77 \times 10^{-5}$ days per year. The longer the orbital period, the more rapidly the period decreases. Following Stepien (1995), the period decreasing rate due to the angular momentum loss (AML) caused by magnetic wind is computed for each system. A comparison shows that the observed $\mathrm{d} P / \mathrm{d} t$ 's are obviously higher than that of the theoretical predictions by 1-3 orders of magnitude. It suggests that the magnetic wind is not likely the determinant mechanism driving the AML in close binaries.
\end{abstract}

Keywords. Detached active binaries, period decreases, magnetic wind.

\section{Introduction}

Angular momentum loss (AML) plays an important role in the evolution of binary stars. AML via magnetized wind is usually thought as the main mechanism for late-type close binaries. Applying the observational results of spin-down of single stars, Stepien (1995) had deduced a formula to compute the AML via magnetic wind in close binary systems. With which he had given a good interpretation on the frequencies and formation time-scale of contact systems evolved from detached binaries. However, a comparison study of this formula to the virtual AML observed in detached late-type binaries is still lack. We report here a period study of active detached binaries. The results are discussed comparison with Stepien's model.

\section{The working sample and data}

A large number of detached late-type binaries which have definite physical parameters (masses and radii) were compiled as the preliminary candidates. Based on the data collected by the database of $B B S A G, A A V S O$ and $V A R$. ASTRO, the behavior of period changes of all these candidates were surveyed. 17 systems, which have sufficient observed times of light minima and present strict long-term orbital period decreases, were finally included in the program sample. The basic data of the 17 stars were given in Table 1 . The times of minima used for period study are mainly taken from the two database mentioned above.

$\dagger$ Present address: National Astronomical Observatories, A20 Datun Road,Chaoyang District,Beijing, 10012,China. 
Table 1. Physical parameters of the six CABS and orbital period decrease rates

\begin{tabular}{lccccccc}
\hline Stars & $\begin{array}{c}M_{1} \\
\left(M_{\odot}\right)\end{array}$ & $\begin{array}{c}M_{2} \\
\left(M_{\odot}\right)\end{array}$ & $\begin{array}{c}R_{1} \\
\left(R_{\odot}\right)\end{array}$ & $\begin{array}{c}R_{2} \\
\left(R_{\odot}\right)\end{array}$ & $\begin{array}{c}\text { Orbital Period } \\
(\text { days })\end{array}$ & $\begin{array}{c}\mathrm{d} P / \mathrm{d} t(\text { Obs }) \\
(\text { days } / y r)\end{array}$ & $\begin{array}{c}\mathrm{d} P / \mathrm{d} t(\text { Com }) \\
(\text { days } / y r)\end{array}$ \\
\hline RT And & 1.230 & 0.910 & 0.92 & 1.26 & 0.62892820 & $-5.15 \times 10^{-8}$ & $-3.38 \times 10^{-10}$ \\
WY Cnc & 1.071 & 0.530 & 0.58 & 0.93 & 0.82936767 & $-8.66 \times 10^{-8}$ & $-1.81 \times 10^{-10}$ \\
MM Her & 1.270 & 1.199 & 2.89 & 1.56 & 7.96032045 & $-7.96 \times 10^{-7}$ & $-6.08 \times 10^{-10}$ \\
RT Lac & 1.482 & 0.600 & 4.81 & 4.41 & 5.07392578 & $-1.29 \times 10^{-6}$ & $-3.88 \times 10^{-9}$ \\
UV Psc & 0.991 & 0.758 & 0.83 & 1.11 & 0.86104648 & $-3.18 \times 10^{-8}$ & $-2.75 \times 10^{-9}$ \\
BH Vir & 1.020 & 1.001 & 1.11 & 1.25 & 0.81687110 & $-1.07 \times 10^{-8}$ & $-3.78 \times 10^{-10}$ \\
AR Lac & 1.260 & 1.130 & 2.72 & 1.52 & 1.98315308 & $-1.20 \times 10^{-6}$ & $-9.02 \times 10^{-10}$ \\
AR Mon & 2.613 & 0.800 & 10.8 & 14.2 & 21.0710678 & $-2.96 \times 10^{-5}$ & $-1.22 \times 10^{-8}$ \\
CM Dra & 0.231 & 0.213 & 0.23 & 0.25 & 1.26838898 & $-4.57 \times 10^{-7}$ & $-3.70 \times 10^{-11}$ \\
ER Vul & 1.099 & 1.051 & 1.08 & 1.11 & 0.69809484 & $-3.05 \times 10^{-9}$ & $-3.28 \times 10^{-10}$ \\
RS Cvn & 1.440 & 1.380 & 4.00 & 1.99 & 4.79767597 & $-2.84 \times 10^{-6}$ & $-1.21 \times 10^{-9}$ \\
RU Cnc & 1.470 & 1.460 & 4.90 & 1.90 & 10.1729706 & $-1.91 \times 10^{-6}$ & $-1.26 \times 10^{-9}$ \\
SS Cam & 1.832 & 1.748 & 6.40 & 2.20 & 4.82353386 & $-2.67 \times 10^{-6}$ & $-2.38 \times 10^{-9}$ \\
SZ Psc & 1.620 & 1.241 & 5.10 & 1.50 & 3.96571971 & $-5.70 \times 10^{-6}$ & $-2.02 \times 10^{-8}$ \\
V471 Tau & 0.760 & 0.740 & 0.83 & 0.01 & 0.52118310 & $-1.38 \times 10^{-9}$ & $-1.34 \times 10^{-10}$ \\
VV Mon & 1.498 & 1.412 & 6.20 & 1.80 & 6.05033577 & $-2.39 \times 10^{-6}$ & $-2.33 \times 10^{-9}$ \\
Z Her & 1.554 & 1.310 & 2.73 & 1.85 & 3.99279951 & $-1.59 \times 10^{-7}$ & $-7.15 \times 10^{-10}$ \\
\hline
\end{tabular}

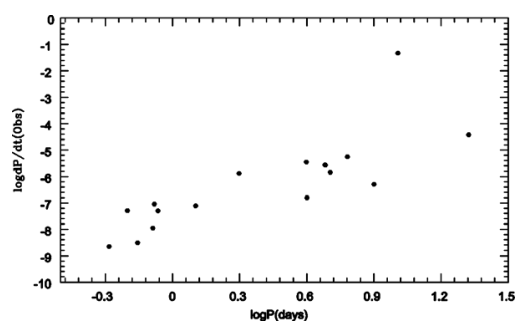

Figure 1. The observed $\mathrm{P}-\mathrm{d} P / \mathrm{d} t$ relation.

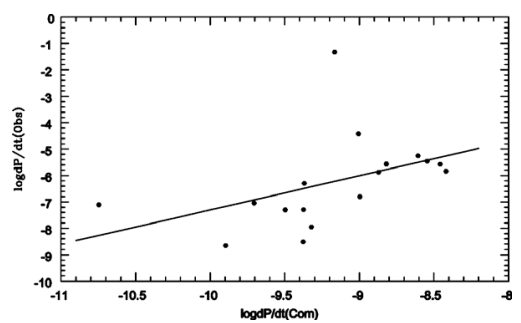

Figure 2. A comparison of the period changing rate between observations and calculations.

\section{Results and Conclusions}

The results of the period analysis for the 17 systems are given in Table 1 . The predicted period changing rate with respect to the magnetic wind computed for each star is also given. Figure 1 represents a relation of the observed $\mathrm{dP} / \mathrm{dt}$ vs. P. It shows that the period decreasing rate seems to be decrease with time, i.e., the longer the period is, the more rapidly the period decreases. This may be caused by some instabilities due to the strong interactions between the components among the short-period systems. A comparison between the observed period decreasing rates and the calculated values is shown in Figure 2. It shows that the observed $\mathrm{dP} / \mathrm{dt}$ are obviously higher than the calculated values by 1-3 orders of magnitude. It is suggested that the magnetic wind seems not to be the only factor for AML.

\section{Acknowledgements}

This work is supported by the Natural Science Foundation of China through grants 10778719, $10573022 \& 10773015$. 